\title{
Características evolutivas dos artigos científicos publicados nos Arquivos Brasileiros de Oftalmologia entre os anos de 1986 e 2000
}

\author{
Evolutive characteristics of the scientific articles published in the Arquivos \\ Brasileiros de Oftalmologia" between 1986 and the year 2000
}

\author{
Antonio Guilherme Gonsalves de Melo Ventura ${ }^{1}$ \\ Aurélio José Gonsalves de Melo Ventura ${ }^{2}$ \\ Saulo André dos Santos ${ }^{3}$
}

Trabalho realizado no Centro Oftalmológico da Paraíba - COP - Campina Grande - Paraíba (PB) - Brasil.

${ }^{1}$ Oftalmologista do Centro Oftalmológico da Paraíba Paraíba (PB) - Brasil.

${ }^{2}$ Oftalmologista do Centro Oftalmológico da Paraíba Paraíba (PB) - Brasil.

${ }^{3}$ Professor de Estatística da Faculdade do Vale do Ipojuca - FAVIP - Caruaru (PE); Coordenador do Setor de Estatística do SESI/SENAI (PB); Professor de Estatística da UNESC - Paraíba (PB) - Brasil.

Endereço para correspondência: Centro Oftalmológico da Paraíba - COP. Rua Afonso Campos, 162 - Centro Campina Grande (PB) CEP 58100-600

E-mail: agventura@hotmail.com

Recebido para publicação em 01.04.2007

Última versão recebida em 02.06.2008

Aprovação em 16.06.2008

\begin{tabular}{|l|}
\hline RESUMO \\
\hline Objetivos: 1) Verificar o número médio de autores por artigo e sua pro- \\
cedência geográfica; 2) Avaliar o idioma das publicações; 3) Avaliar as \\
referências bibliográficas quanto ao número das mesmas por artigo, tipo \\
e revistas mais citadas, além do comportamento do crescimento das \\
referências ao longo dos anos. Métodos: Foi realizada revisão sistemática \\
de 876 publicações dos Arquivos Brasileiros de Oftalmologia (ABO), no \\
período de janeiro de 1986 a dezembro do ano 2000, divididos em três \\
grupos qüinqüenais para comparação temporal. Resultados: O número \\
médio de autores por artigo foi de 3,73 $\pm 1,7$, sem variação significante. \\
Quanto a procedência geográfica nacional, a região Sudeste foi respon- \\
sável por 83,6\% das publicações. O idioma de publicação mais prevalente \\
foi o português (94,7\%). O número de referências por artigo foi 12,7 7,5 \\
e revista científica (81,8\%) foi o tipo documental mais referido. American \\
Journal of Ophthalmology, Archives of Ophthalmology e Ophthalmology \\
foram as revistas mais citadas, com aumento progressivo ( $\left.\mathrm{R}^{2}=0,6075\right)$, \\
assim como também das revistas nacionais ( $\left.\mathrm{R}^{2}=0,7861\right)$. Conclusões: \\
Durante o período investigado, o número médio de autores por artigo não \\
apresentou variação significativa e sua procedência geográfica foi, na \\
maioria, a região Sudeste. O idioma português foi o mais freqüentemente \\
utilizado. O documento citado majoritariamente foi revista científica. As \\
citações, tanto nacionais quanto estrangeiras, tiveram crescimento signi- \\
ficativo.
\end{tabular}

Descritores: Publicações periódicas; Artigo de revista; Indexação; Base de dados; Fator de impacto

\section{INTRODUÇÃO}

Os periódicos ganharam nos últimos anos uma importância inequívoca na Medicina $^{(1)}$, e representam o meio de informação mais democrático e confiável, uma vez que adotam, em sua maioria, critérios específicos, seletivos e rigorosos para publicação de artigos científicos ${ }^{(2)}$.

Um bom indicador do potencial científico de um país é constituído pelas publicações de seus artigos e da difusão de suas investigações em revistas, tanto de âmbito nacional, quanto internacional ${ }^{(3-4)}$.

Neste contexto, o fator de impacto (número de citações em relação ao número de publicações na revista nos dois anos anteriores) é relevante para a reputação do periódico e pode ser entendido como fator de qualidade $^{(5)}$. 
Por conseguinte, para que uma publicação tenha um aceitável fator de impacto deve ser lida, consultada e, no mínimo, citada por determinadas revistas, indexadas em órgãos de informações (base de dados) ${ }^{(6)}$.

A aspiração de toda revista periódica é tornar-se indexada (influenciada pelas Universidades e pelas agências de fomento à pesquisa) ${ }^{(7)}$, buscando, desta forma, o reconhecimento e a pretensão de aumentar o alcance da divulgação e exposição da ciência ${ }^{(8)}$.

O presente estudo busca avaliar os Arquivos Brasileiros de Oftalmologia (ABO), órgão oficial do Conselho Brasileiro de Oftalmologia (CBO), com publicação ininterrupta desde 1938, periódico que segue os principais critérios de submissão editorial sugeridos pelo "International Committee of Medical Journal Editors", possuindo "peer review" e publicando artigos em português, inglês e espanhol ${ }^{(7)}$, representando assim, o veículo de divulgação da atividade científica da comunidade oftalmológica brasileira de maior impacto ${ }^{(2)}$ e que já encontra-se indexada nas bases de dados: LILACS, periódica, MEDLINE e EMBASE.

Tendo em vista as transformações evolutivas dos artigos científicos, este artigo tem por objetivos: verificar o número médio de autores por artigo; avaliar a procedência geográfica dos autores, além da natureza jurídica da instituição, quando nacional; avaliar o idioma de publicação, assim como os assuntos mais abordados; avaliar as referências bibliográficas quanto ao número das mesmas por artigo, tipo e revistas mais citadas, além do comportamento do crescimento destas referências ao longo dos anos.

\section{MÉTODOS}

Foi realizada revisão sistemática, sem metanálise, dos artigos científicos publicados nos $\mathrm{ABO}$, no período de janeiro de 1986 a dezembro de 2000, utilizando o CD-ROM editado pelo CBO em 2001 ${ }^{(9)}$, e distribuído no XXXI Congresso Brasileiro de Oftalmologia.

Critérios de Inclusão: foram incluídos artigos originais e relatos de caso, publicados na íntegra pelos $\mathrm{ABO}$, entre os anos de 1986 a 2000.

Critérios de Exclusão: não foram incluídos na avaliação os seguintes formatos de publicação: Cartas ao Editor, Editorial, Anais de Congresso, Revisão Temática, Atualização Continuada e Resumo de artigos publicados no exterior.

Foram publicados 4.253 estudos, em 90 fascículos, durante os 15 anos analisados. Aplicando-se os critérios de inclusão e de exclusão, chegou-se a amostra final de 876 artigos, correspondendo a $20,5 \%$ do número total publicado de 1986 a 2000 .

Foram reconhecidas as seguintes variáveis de cada artigo: número de autores; procedência geográfica, caracterizadas como nacionais e internacionais e, dentre as nacionais, a região de origem (Norte, Nordeste, Centro-Oeste, Sudeste e Sul); natureza jurídica da instituição, considerando-se entre as instituições nacionais se a mesma é pública (estadual ou federal), filantrópica, particular ou outros (organizações nãogovernamentais, secretarias de saúde, conselhos de classe, etc); idioma de publicação; temas mais abordados; número de referências bibliográficas por artigo; tipo de referências bibliográficas, classificadas segundo critérios pré-estabelecidos em referência nacional ou estrangeira, revistas científicas ou publicações de caráter periódico, livros, teses de mestrado ou doutorado, outros (incluindo monografias sobre temas específicos publicados por universidades, laboratórios farmacêuticos ou organizações científicas, comunicações em congressos, comunicações pessoais, folhetos, catálogos, etc), periódicos mais citados, dentre nacionais e internacionais ${ }^{(3)}$.

Vale ressaltar que, quando mais de uma instituição era responsável pela pesquisa foi considerada a procedência e a natureza jurídica da qual o primeiro autor fazia parte no momento do estudo.

Para posterior avaliação das características evolutivas dos $\mathrm{ABO}$, as informações colhidas foram divididas em três grupos qüinqüenais, a saber: Grupo I - artigos publicados de 1986 a 1990; Grupo II - artigos publicados de 1991 a 1995 e Grupo III artigos publicados de 1996 a 2000.

Para avaliação dos resultados, foi utilizada estatística descritiva nas observações de freqüências absolutas e relativas. Os dados foram transferidos para planilha eletrônica Excel. A análise estatística foi realizada utilizando o Pacote Estatístico SPSS 13.0 (Statistical Package for Social Science - for Windows) e os testes do qui-quadrado, análise de regressão e coeficiente de determinação de Pearson. Foram considerados valores significativos com $\mathrm{p} \leq 0,05$.

\section{RESULTADOS}

Aplicando-se os critérios para o estudo, foram analisados 876 artigos, divididos em três períodos de cinco anos (grupos qüinqüenais).

Desta forma, avaliando-se os 876 artigos, o número de autores por artigo, foi de $3,73 \pm 1,7$. Não se observou nenhuma variação estatisticamente significativa no número médio de autores ao longo dos períodos qüinqüenais $\left(X^{2}=3,58\right.$, ou seja, $p>0,05$ ) (Tabela 1 ).

De acordo com a procedência geográfica, 37 (4,2\%) artigos eram estrangeiros e $839(95,7 \%)$ nacionais. Quanto à procedência regional brasileira, nos três períodos quinqüenais, a maioria dos artigos originou-se da região Sudeste, com 422 $(83,6 \%)$ publicações, seguindo-se da região Sul, com $89(10,6 \%)$ e da região Nordeste, com 26 (3\%) das publicações.

O tipo de Instituição também se diferenciou, no que tange à quantidade de artigos, e as instituições públicas foram responsáveis pela autoria de $664(79,1 \%)$ artigos, valor expressivamente maior do que as instituições filantrópicas e privadas, responsáveis por 101 (12\%) e 67 (8\%) publicações, respectivamente. 
O idioma de publicação mais freqüente foi o português, com $830(94,7 \%)$ estudos publicados nesta língua, seguido pelo inglês, com 41 (4,6\%) e o espanhol, com $5(0,6 \%)$ estudos.

Dentre os temas mais abordados na publicação de artigos, cinco foram encontrados com maior freqüência, se responsabilizando por $60,5 \%$ do total dos temas. As áreas mais citadas foram córnea e doenças externas $(179 / 20,4 \%)$, seguida de retina e vítreo $(124 / 14,1 \%)$, glaucoma $(111 / 12,6 \%)$, uveíte $(68 / 7,7 \%)$ e catarata e lentes intra-oculares $(50 / 5,7 \%)$.

As 876 publicações continham 11.155 referências e o terceiro qüinqüênio (Grupo III - 1996 a 2000) foi o responsável pelo maior número delas. $\mathrm{O}$ número médio de referências por artigo foi de $12,78 \pm 7,5$. O tipo documental referido majoritariamente foi a revista científica, com 9.135 (81,8\%) citações, sendo $1.108(9,9 \%)$ de periódico nacional e $8.027(71,9 \%)$ de estrangeiro, seguido do livro com $1.576(14,1 \%)$ citações (Tabela 2).

Analisando-se as 9.135 referências que correspondiam a revistas periódicas (nacionais e estrangeiras), durante o período de estudo, encontraram-se 928 revistas diferentes. Literalmente 10 revistas de oftalmologia que representavam $1,0 \%$ do total de revistas (10/928) apareciam em 55,7\% das referências deste tipo (5095/9135). Estes periódicos foram: American Journal of Ophthalmology, Archives of Ophthalmology, Ophthalmology, Arquivos Brasileiros de Oftalmolo-

\begin{tabular}{|c|c|c|c|c|c|}
\hline \multirow{2}{*}{$\begin{array}{l}\text { Grupos } \\
\text { qüinqüenais }\end{array}$} & \multicolumn{2}{|c|}{ Artigos } & \multicolumn{2}{|c|}{ Autores } & \multirow{2}{*}{$\begin{array}{c}\text { Índice de } \\
\text { autores/artigo* }\end{array}$} \\
\hline & $n \div$ & $\%$ & $\overline{n^{\circ}}$ & $\%$ & \\
\hline $\begin{array}{l}\text { Grupo I } \\
(1986 \text { - 1990) }\end{array}$ & 223 & 25,4 & 767 & 23,4 & $3,43 \pm 1,5$ \\
\hline $\begin{array}{l}\text { Grupo II } \\
(1991-1995)\end{array}$ & 264 & 30,1 & 936 & 28,6 & $3,54 \pm 1,6$ \\
\hline $\begin{array}{l}\text { Grupo III } \\
(1996-2000)\end{array}$ & 389 & 44,4 & 1568 & 47,9 & $4,03 \pm 1,7$ \\
\hline Total & 876 & 100 & 3271 & 100 & $3,73 \pm 1,7$ \\
\hline
\end{tabular}

gia, British Journal of Ophthalmology, Investigative Ophthalmology \& Visual Science, Revista Brasileira de Oftalmologia, Survey of Ophthalmology, Acta Ophthalmologica e Ophthalmic Surgery (Tabela 3 ).

Ao se comparar a freqüência de citações das referências estrangeiras (American Journal of Ophthalmology, Archives of Ophthalmology e Ophthalmology) e nacionais (ABO e Revista Brasileira de Oftalmologia) mais citadas, observouse tendência linear ascendente, tanto para as estrangeiras $\left(\mathrm{R}^{2}=0,6075\right)$, quanto para as nacionais $\left(\mathrm{R}^{2}=0,7861\right)$, com crescimento significativo para ambas $(\mathrm{p}=0,001)$ (Gráfico 1$)$.

\section{DISCUSSÃO}

A análise de 876 artigos originais e relatos de casos publicados nos $\mathrm{ABO}$, entre 1986 e 2000, demonstrou que não houve diferença significativa no tocante ao número de autores por artigo ao longo dos anos, resultado semelhante ao encontrado em outro estudo no qual foram avaliados 10 anos de publicação ${ }^{(4)}$. Contudo, em artigos nos quais foram avaliados 30 anos ou mais, observou-se aumento gradativo e estatisticamente significativo do número de autores por artigo ${ }^{(5,10)}$, o que poderia se justificar pelo fato da revista científica ter se tornado a fonte principal de exposição dos autores e da necessidade de formação de grupos de trabalho cada vez maiores, pela crescente complexidade da investigação científica.

Quanto à procedência regional brasileira, a afiliação do primeiro autor revelou uma distribuição desproporcional durante os períodos qüinqüenais, com participação expressiva (mais de $80 \%$ da produção nacional) situada na região Sudeste, fato também constatado em outros estudos ${ }^{(5,11)}$, provavelmente explicado pela maior concentração de instituições médicas e de profissionais, além da centralização econômica do país neste eixo geográfico, o que poderia refletir maiores subsídios para pesquisa nesta região.

O tipo de instituição geradora da pesquisa foi predominantemente pública, fato também evidenciado por outro trabalho realizado em $1999^{(11)}$, que revelou um dado complementar importante: "Os dados apontam para instituições que man-

\begin{tabular}{|c|c|c|c|c|c|c|c|c|c|c|c|c|c|c|c|}
\hline \multirow{4}{*}{$\begin{array}{l}\text { Grupos } \\
\text { qüinqüenais }\end{array}$} & & & \multirow{4}{*}{$\begin{array}{l}\text { Referên- } \\
\text { cias/artigo* }\end{array}$} & \multicolumn{12}{|c|}{ Tipos de documentos } \\
\hline & \multirow{2}{*}{\multicolumn{2}{|c|}{ Referências }} & & \multicolumn{4}{|c|}{ Revista científica } & \multirow{2}{*}{\multicolumn{2}{|c|}{ Livro }} & \multirow{2}{*}{\multicolumn{2}{|c|}{ Anais cong. }} & \multirow{2}{*}{\multicolumn{2}{|c|}{ Tese }} & \multirow{2}{*}{\multicolumn{2}{|c|}{ Outros }} \\
\hline & & & & \multicolumn{2}{|c|}{ Nacional } & \multicolumn{2}{|c|}{ Estrangeira } & & & & & & & & \\
\hline & $\mathrm{n}^{\circ}$ & $\%$ & & $n \circ$ & $\%$ & $\mathrm{n}$ 우 & $\%$ & \multicolumn{2}{|c|}{$\mathrm{n} \div \quad \%$} & $\mathrm{n}$ 은 & $\%$ & \multicolumn{2}{|c|}{ nㅇ $\%$} & & $\%$ \\
\hline $\begin{array}{l}\text { Grupo I } \\
(1986 \text { - 1990) }\end{array}$ & 3071 & 27,5 & $13,77 \pm 7,7$ & 231 & 7,5 & 2113 & 68,8 & 576 & 18,7 & 88 & 2,8 & 33 & 1,0 & 30 & 0,9 \\
\hline $\begin{array}{l}\text { Grupo II } \\
(1991-1995)\end{array}$ & 3128 & 28,0 & $11,84 \pm 7,4$ & 305 & 9,7 & 2291 & 73,2 & 418 & 13,3 & 45 & 1,4 & 28 & 0,8 & 41 & 1,3 \\
\hline $\begin{array}{l}\text { Grupo III } \\
(1996-2000)\end{array}$ & 4956 & 44,4 & $12,74 \pm 7,5$ & 572 & 11,5 & 3623 & 73,1 & 582 & 11,7 & 47 & 0,9 & 52 & 1,0 & 80 & 1,6 \\
\hline Total & 11155 & 100 & $12,78 \pm 7,5$ & 1108 & 9,9 & 8027 & 71,9 & 1576 & 14,1 & 180 & 1,6 & 113 & 1,0 & 151 & 1,3 \\
\hline
\end{tabular}




\begin{tabular}{|c|c|c|c|c|c|c|c|c|}
\hline \multirow[t]{2}{*}{ Revistas } & \multicolumn{2}{|c|}{ Grupo I } & \multicolumn{2}{|c|}{ Grupo II } & \multicolumn{2}{|c|}{ Grupo III } & \multicolumn{2}{|c|}{ Total } \\
\hline & $\mathrm{n}^{\circ}$ & $\%$ & $n \div$ & $\%$ & $n \circ$ & $\%$ & n은 & $\%$ \\
\hline Archives of Ophthalmology & 300 & 12,7 & 317 & 12,2 & 455 & 10,8 & 1072 & 11,7 \\
\hline Ophthalmology & 88 & 3,7 & 227 & 8,7 & 437 & 10,4 & 752 & 8,2 \\
\hline Arquivos Brasileiros de Oftalmologia & 117 & 5,0 & 168 & 6,4 & 340 & 8,1 & 625 & 6,8 \\
\hline $\begin{array}{l}\text { Investigative of Ophthalmology } \\
\text { \& Visual Science }\end{array}$ & 69 & 3,0 & 82 & 3,1 & 156 & 3,7 & 307 & 6,1 \\
\hline Revista Brasileira de Oftalmologia & 55 & 2,3 & 81 & 3,1 & 163 & 3,8 & 299 & 3,2 \\
\hline Survey of Ophthalmology & 27 & 1,1 & 58 & 0,7 & 68 & 1,6 & 153 & 1,6 \\
\hline Acta Ophthalmologica & 40 & 1,7 & 46 & 1,7 & 59 & 1,4 & 145 & 1,5 \\
\hline Ophthalmic Surgery & 21 & 0,8 & 44 & 1,7 & 75 & 1,7 & 140 & 1,5 \\
\hline
\end{tabular}

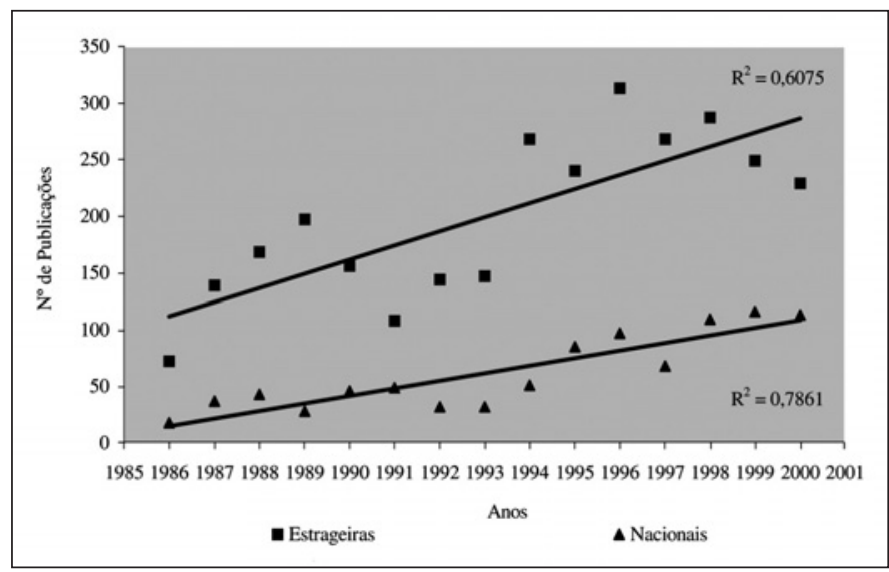

Gráfico 1 - Regressão linear das revistas estrangeiras (American Journal of Ophthalmology, Archives of Ophthalmology e Ophthalmology) e nacionais (Arquivos Brasileiros de Oftalmologia e Revista Brasileira de Oftalmologia) mais citadas e utilizadas como referências dos artigos publicados nos Arquivos Brasileiros de Oftalmologia

têm programas de pós-graduação, sugerindo a correlação entre existência dos programas e produção". No entanto, na pesquisa atual, não se procurou avaliar se as instituições possuíam ou não tais programas.

$\mathrm{O}$ idioma das publicações nos ABO foi predominantemente o português nos três grupos qüinqüenais e a língua inglesa correspondeu a uma pequena parcela $(4,6 \%)$ do total dos artigos, assemelhando-se a outra pesquisa nacional que demonstrou $2,8 \%$ do total de periódicos publicados em inglês e $95,7 \%$, em português ${ }^{(5)}$. A publicação em português foi indicada como o principal fator de discriminação ${ }^{(5)}$, entre as imposições que órgãos anglo-saxônicos estabelecem para suas seleções ${ }^{(7)}$ e, portanto, seus autores são menos citados do que aqueles que publicam em inglês, língua franca do mundo científico atual ${ }^{(1)}$. Não obstante, nossa língua mãe não foi obstáculo para indexação dos $\mathrm{ABO}$ em bases de dados referenciais internacionais aumentando o alcance de propagação e exposição da oftalmologia brasileira. De fato, há 35 anos as instruções para autores dos ABO permitem a submissão de artigos em inglês como forma de atrair artigos internacionais para publicação sendo, contudo, a escolha do idioma opção dos autores e não do conselho editorial ${ }^{(12)}$.

Em 2005, após a indexação dos ABO em bases de dados de prestígio mundial, provavelmente pela vitrine que se abriu expondo a produção oftalmológica brasileira à leitura internacional, mais autores iriam preferir publicar seus trabalhos no modo a que fossem apreciados na íntegra, não apenas por seus resumos e presumiu-se que pudesse haver aumento dos artigos dos ABO publicados em Inglês de forma livre e espontânea ${ }^{(13)}$. Isto realmente ocorreu, subindo o número de artigos publicados nesse idioma de 15 em 2005 para 30 em $2006^{(12)}$.

As cinco áreas temáticas da oftalmologia que foram mais abordadas foram responsáveis por $60,5 \%$ do total de artigos, aparentemente esclarecido por serem áreas que oferecem maior número de vagas em cursos de especialização, resultando em grupos de estudos mais amplos, além de serem áreas que têm apresentado maior evolução, tanto clínica, quanto cirúrgica.

A avaliação das 11.155 referências bibliográficas evidenciou um número médio das mesmas por artigo constante ao longo dos anos, resultado análogo ao encontrado em outro estudo, porém, com a média mais elevada $(25,2$ referências por $\operatorname{artigo}^{(13)}$, ao passo que, durante o período de 30 anos investigado em $1994^{(10)}$, foi demonstrado aumento progressivo no número de referências por artigo. Vale salientar que o comitê editorial dos ABO não impõe um número limite de referências bibliográficas, assim como a maioria dos comitês de periódicos nacionais e internacionais.

O processo de comunicação se transmitiu para os artigos científicos, na forma de referências, primordialmente através das revistas $(81,8 \%)$, seguido pelos livros $(14,1 \%)$, coincidindo com outras publicações ${ }^{(4,10,14-15)}$. A facilidade na busca por informações em bases de dados permitiu uma exposição ampla e atualizada de pesquisas científicas completas utilizadas como referências, passando os livros a adquirir uma importância secundária e com influência regressiva, como revelado também no presente estudo. 
Quanto à dispersão bibliográfica, as 928 revistas distintas (nacionais e estrangeiras) forneceram 9.135 referências, das quais, $55,7 \%$ correspondiam a apenas $1 \%$ do total das revistas (10 periódicos), incluindo duas nacionais (ABO e Revista Brasileira de Oftalmologia). Apesar de suas influências, o pesquisador brasileiro cita menos publicações de seu próprio país, já que as três revistas com referências mais citadas são norte-americanas (American Journal of Ophthalmology, Archives of Ophthalmology e Ophthalmology). Fato semelhante também ocorreu em outras pesquisas ${ }^{(4,10)}$, gerando, como conseqüência, menor índice de fator de impacto para as publicações nacionais.

A tendência linear das citações apresentou ascensão, tanto para as referências estrangeiras, quanto para as nacionais. Contudo, as nacionais demonstraram menor dispersão de dados que as estrangeiras e seu coeficiente de determinação de Pearson $\left(\mathrm{R}^{2}=0,7861\right)$ relação mais forte que as estrangeiras $\left(\mathrm{R}^{2}=0,6075\right)$, com crescimento mais consistente. Por outro lado, em 2003, observou-se linha de tendência linear descendente para citações estrangeiras e ascendente para citações nacionais ${ }^{(3)}$, representando sinal de prestígio da revista nacional como fonte de referência entre os autores brasileiros.

Do período de 1986 a 2000 os ABO evidenciaram citações predominantemente estrangeiras. Em 2004, autor brasileiro comenta que "citar os ABO bibliograficamente melhora sua qualificação junto às bases de dados internacionais, aumentando seu fator de impacto"(8).

Publicações nos $\mathrm{ABO}$ terão repercussão semelhante às revistas estrangeiras consolidadas, já que as indexações são as mesmas.

Por fim, sugere-se a realização de estudos de avaliação de publicações subseqüentes ao ano de 2000, compreendendo o período de ingresso dos $\mathrm{ABO}$ em grandes bases de dados de referência internacional e sua influência no meio científico nacional.

\section{CONCLUSÕES}

Este estudo demonstrou que, durante o período investigado, não houve variação significativa no número médio de autores por artigo e que sua procedência geográfica foi, na maioria, oriunda da região Sudeste, assim como, a instituição pública, a responsável pelo número expressivamente maior de publicações. Medidas de apoio e estímulo à produção científica realizada pelos centros de ensino em oftalmologia, poderiam disseminar os conhecimentos gerados e atenuar o desequilíbrio regional.

Outro aspecto evidenciado foi a prevalência do idioma português e da área córnea e doenças externas, como assunto mais abordado, nas publicações. Já, quanto às referências, o tipo documental citado majoritariamente foi a revista científica, representada primeiramente pelas de origem norte-americana. As citações, tanto nacionais quanto estrangeiras, tiveram crescimento significativo ao longo do período avaliado.

\section{AGRADECIMENTOS}

Agradecemos ao Dr. Giovannini Cesar Figueiredo pelo estímulo à realização deste estudo e ao comitê de ética em pesquisa da Fundação Altino Ventura pela sua avaliação.

\section{ABSTRACT}

Purpose: 1) To verify the average number of the authors per article and its geographic origin; 2) To evaluate the language of publications; 3) To evaluate the bibliographical references with their number per article, type and most cited periodicals, besides the behavior of the growth of the references along the years. Methods: Systematic revision was done of 876 publications of Arquivos Brasileiros de Oftalmologia (ABO) in the period of January of 1986 to December of the year 2000. These scientific publications were separated in to three quinquennial groups. Results: The average number of authors per article was $3.73 \pm 1.7$. According to the geographic origin, $83.6 \%$ of the publications came from the Southeastern region. Portuguese was the language of the majority of articles $(94.7 \%)$. The number of references per article was $12.7 \pm 7.5$, and a scientific periodical (81.8\%) was the most reported document. American Journal of Ophthalmology, Archives of Ophthalmology and Ophthalmology were the most cited periodicals and their growth $\left(\mathrm{R}^{2}=0.6075\right)$, as well as of the national ones $\left(\mathrm{R}^{2}=0.7861\right)$ was increasing. Conclusion: The average number of authors per article did not present significant variation and the geographical origin was mostly the Southeastern region. The Portuguese language was the most frequent. The mostly quoted document was the scientific periodical. The quotations of international publications as well as national presented a significant increase.

Keywords: Periodicals; Journal article; Indexing; Databases; Impact factor

\section{REFERÊNCIAS}

1. Elkis H. Fatores de impacto de publicações psiquiátricas e produtividade científica. Rev Bras Psiquiatr. 1999;21(4):231-6.

2. Crosta F, Nishiwaki-Dantas MC, Silvino W, Dantas PEC. Diversidade e freqüência dos desenhos de estudos científicos e métodos estatísticos nos Arquivos Brasileiros de Oftalmologia: revisão sistemática dos Arquivos Brasileiros de Oftalmologia - 1993 a 2002. Arq Bras Oftalmol. 2005;68(3):339-45.

3. Figueiredo GC. Freqüência de citação das referências nacionais na Revista Brasileira de Ortopedia em um período de 36 anos. Rev Bras Ortop. 2003;38(7): 410-5.

4. Tortosa Serrano JA, Mulero Cervantes JF, Hernández-Palazon J, García-Cayuela JM. Análisis bibliométrico de los artículos originales publicados em la Revista Española de Anestesiología y Reanimacíon durante 10 años (19871996). Rev Esp Anestesiol Reanim. 1998;45(7):268-74.

5. Mansur AJ, Abud AS, Albuquerque CP. Tendências das publicações nas fases trimestral, bimestral e mensal das cinco décadas dos Arquivos Brasileiros de Cardiologia. Arq Bras Cardiol. 2000;75(1):1-7.

6. Blaso Casares FJ, Pinsach Elías L. Revisión crítica de las referencias bibliográficas. Arch Esp de Urol. 1993;46(1):1-5. 
7. Bicas HEA, Rother ET, Braga MER. Ainda sobre indexações. Arq Bras Oftalmol. 2002;65(1):5-6

8. Dantas PEC. Indexação bibliográfica em bases de dados: O que é? Para que serve? Onde estamos? Arq Bras Oftalmol. 2004;67(4):569-70.

9. Arquivos Brasileiros de Oftalmologia (1986-2000) [CD-ROM]. Conselho Brasileiro de Oftalmologia. Intec-System Tecnologia.. São Paulo; 2001.

10. Pulido M, González JC, Sanz F. Artículos originales publicados en Medicina Clínica durante 30 años (1962-1992): número de autores, intervalo entre la aceptación y publicación y referencias bibliográficas. Med Clin (Barc). 1994;103(20):770-5.

11. Yamamoto $\mathrm{OH}$, Souza CC, Yamamoto ME. A produção científica na psicologia: uma análise dos periódicos brasileiros no período 1990-1997. Psicol Reflex Crit. 1999;12(2):549-65.
12. Muccioli C, Campos M, Goldchmit M, Dantas PEC, Bechara SJ, Costa VP. Artigos em inglês nos Arquivos Brasileiros de Oftalmologia: um resultado da globalização. Arq Bras Oftalmol. 2006;69(4):461.

13. Bicas HEA, Muccioli C, Goldchmit M, Campos MSQ, Dantas PEC, Bechara SJ, et al. Prestigiosa validação de nossos padrões editoriais. Arq Bras Oftalmol. 2005;68(1):5-6.

14. Acea Nebril B, Figueira Moure A, Gómez Freijoso C. Artículos originales publicados em la Revista Española de Enfermedades Digestivas (1993-1998): autoría, demora en la publicación y referencias bibliograficas. Rev Esp Enferm Dig. 2000;92(9):573-85.

15. Aleixandre R, Gimenez Sánchez JV, Terrada ML, López Piñero JM. Análisis del consumo de información en la revista Medicina Clínica. Med Clin (Barc). 1994;103(7):246-51.

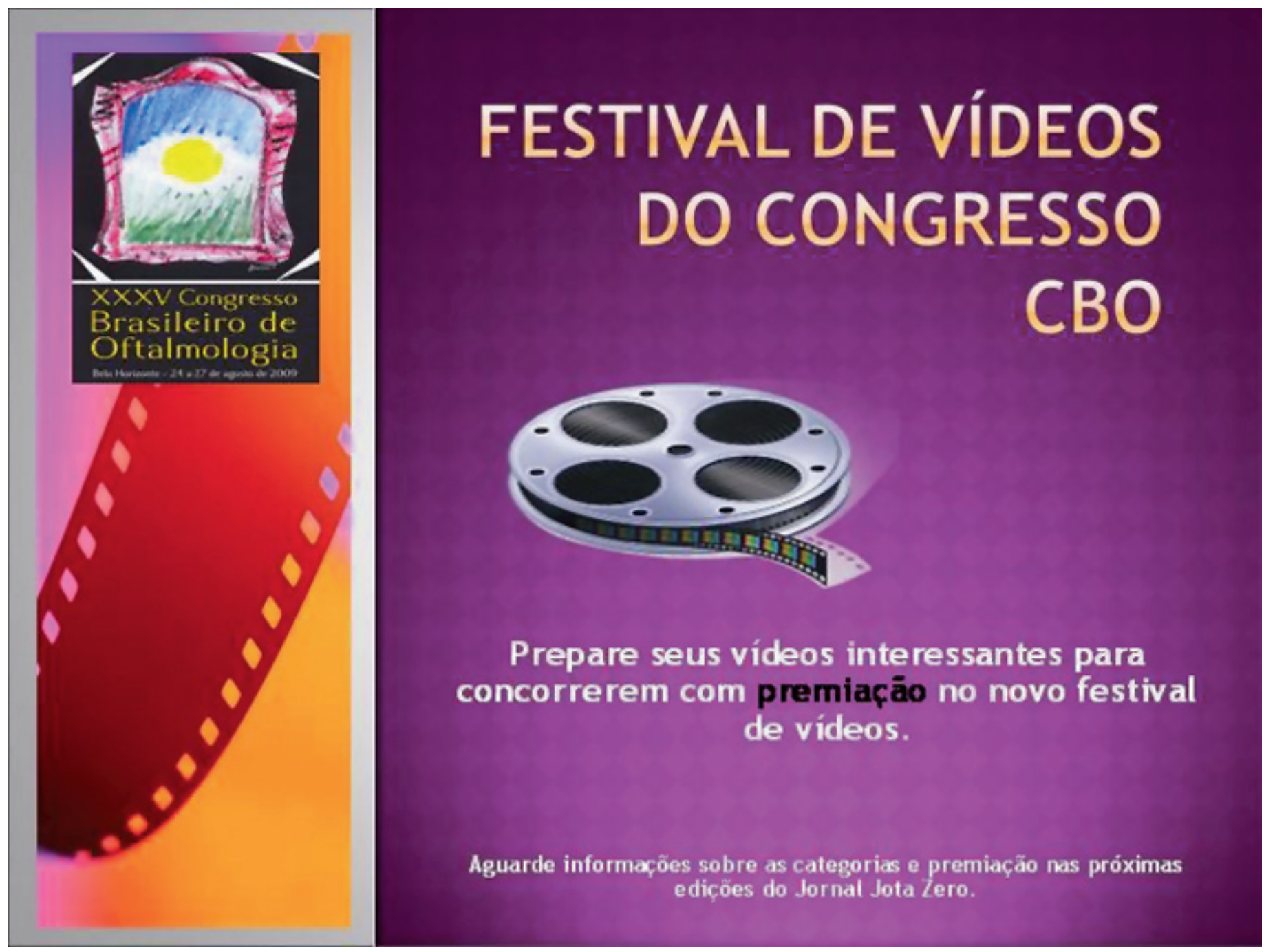

